\title{
ON THE DEFINING FIELD OF A DIVISOR IN AN ALGEBRAIC VARIETY ${ }^{1}$
}

\author{
WEI-LIANG CHOW
}

In the system of algebraic geometry as developed by A. Weil in his recent book, Foundations of algebraic geometry, a variety $U$ in the $n$ space is defined as the set of all equivalent couples $(k, P)$, each consisting of a field $k$ and a point $P$ in the $n$-space such that the field $k(P)$ is a regular extension of $k$. Two such couples $\left(k^{\prime}, P^{\prime}\right)$ and $\left(k^{\prime \prime}, P^{\prime \prime}\right)$ are called equivalent if every finite specialization of $P^{\prime}$ over $k^{\prime}$ is also one of $P^{\prime \prime}$ over $k^{\prime \prime}$ and conversely. Any field $k$ which enters into such a couple is called a field of definition of the variety $U$. It has been shown by Weil in his book that among all the fields of definition of a variety $U$ there is a smallest one which is contained in all of them, which we shall call the defining field of the variety $U$. A $d$-cycle $G$ in a variety $U$ of dimension $r$ is a finite set of simple subvarieties of dimension $d$ in $U$, to each of which is assigned an integer called its multiplicity; a cycle is called positive if the multiplicity of each of its component varieties is positive. Let $K$ be a field of definition of $U$. Then the $G$ is said to be rational over $K$ if it satisfies the following conditions: (1) each component variety of $G$ is algebraic over $K ;(2)$ if a variety is a component of $G$, then all the conjugate varieties over $K$ are also components of $G$ with the same multiplicity; (3) the multiplicity of each component of $G$ is a multiple of its order of inseparability. The question arises whether there is a smallest one among all the fields over which the cycle $G$ is rational. If such a smallest field exists, we shall call it the defining field of the cycle $G$. One observes that since by definition every field over which the cycle $G$ is rational must be a field of definition of the variety $U$, it follows that the defining field of $G$, if it exists, must contain the defining field of $U$.

The following simple example shows that in general a cycle does not have a defining field. Consider the variety $U_{0}$ consisting of the one point $\left(x^{1 / p}, y^{1 / p}\right)$ in the 2 -space $S_{2}$, where $x$ and $y$ are independent variables over a field $k$ of characteristic $p$; this variety $U_{0}$ is a simple subvariety of $S_{2}$. The 0 -cycle $p U_{0}$ is rational over both fields $k\left(x, y^{1 / p}\right)$ and $k\left(x^{1 / p}, y\right)$; but it is not rational over the field $k\left(x, y^{1 / p}\right) \cap k\left(x^{1 / p}, y\right)$ $=k(x, y)$.

In a recent discussion, Weil has communicated to me the conjecture

Received by the editors September 20, 1949.

1 This note is essentially an extract from a letter of the author to Professor Andre Weil, dated February 3, 1949. 
that in case of a divisor in a variety there always exists a defining field. I shall give here a proof of this conjecture, based on the method of the associated forms. ${ }^{2}$ Let $U$ be a variety of dimension $r$ in the $n$-space and let $k$ be its defining field. Let $F\left(Z, Z^{(1)}, \ldots, Z^{(r)}\right)$ be the associated form of $U$, where the $(Z),\left(Z^{(1)}\right), \cdots,\left(Z^{(r)}\right)$ are $r+1$ sets of $n+1$ indeterminates each. It can be easily shown that this form $F\left(Z, Z^{(1)}, \cdots, Z^{(r)}\right)$ is rational over $k$ and absolutely irreducible. We can restrict ourselves to positive cycles in $U$, since every cycle rational over a field $K$ is the difference of two positive cycles which are rational over $K$. To each positive $d$-cycle $G$ in $U$ (since it is also a positive $d$-cycle in the $n$-space) there corresponds an associated form $f\left(Z, Z^{(1)}, \cdots, Z^{(d)}\right)$; let $(f)$ be the set of coefficients of this form. Then the field $k((f))$ is contained in every field over which the cycle $G$ is rational. ${ }^{3}$ It is clear that if the cycle $G$ is rational over $k((f))$, then this field is its defining field. Hence, to prove our conjecture, it is necessary only to show that in case $d=r-1$ the divisor $G$ is rational over the field $k((f))=K$.

Without loss of generality we can assume that the form $f\left(Z, Z^{(1)}, \cdots, Z^{(r-1)}\right)$ is irreducible over $K$; for otherwise we need only to repeat the same argument for each irreducible factor of this form over $K$. Over the algebraic closure of $K$ the form $f\left(Z, Z^{(1)}, \cdots\right.$, $\left.Z^{(r-1)}\right)$ will then be the $p^{e}$ th power of a product of distinct irreducible forms, all conjugate with respect to each other over $K$; this $p^{e}$ is by definition the multiplicity of each component of the divisor $G$. Let $(x)$ be a generic point of one of the components of $G$ over $K$ (it does not matter which, as they are all conjugates over $K$ ). We have to prove that the field $K(x)$ has the order of inseparability $\leqq p^{e}$ over $K$.

Let $z_{j}^{(i)}, i=1, \cdots, r, j=1, \cdots, n$, be $n r$ independent variables over $K(x)$, and set $z_{0}^{(i)}=-\sum_{j=1}^{n} x_{j} z_{j}^{\left(i_{j}\right.}$. Since the point $(x)$ has the dimension $r-1$, the $r(n+1)-1$ elements $z_{j}^{(i)}$, for all $i, j$ except $i=r$, $j=0$, are independent variables over $K$. Hence, the form $f\left(Z, z^{(1)} \cdots\right.$, $\left.z^{(r-1)}\right)$ is exactly the $p^{e}$ th power of a product of distinct linear forms. Let $f\left(Z_{0}\right)$ be the polynomial obtained from $f\left(Z, z^{(1)}, \cdots, z^{(r-1)}\right)$ by

${ }^{2}$ See Chow and van der Waerden, Math. Ann. vol. 113 (1937) pp. 692-704. Strictly speaking, the concept of the associated form is defined only for varieties and positive cycles in a projective space. However, since the affine $n$-space can be extended to a projective space and every variety in the former can be extended to a variety in the latter in a unique way, it is easily seen that our application of this concept to varieties and positive cycles in the affine $n$-space is justified.

${ }^{3}$ If $(f)=\left(f_{0}, f_{1}, \cdots, f_{t}\right)$ is a set of quantities, not all zero, then the field $K((f))$ is the extension of $K$ obtained by the adjunction of all the ratios of any two non-vanishing elements of the set $(f)$. 
setting $Z_{j}=z_{j}^{(r)}, j=1, \cdots, n$; then $f\left(Z_{0}\right)$ is the $p^{e}$ th power of a separable polynomial. Since $z_{0}^{(r)}$ is a root of this polynomial $f\left(Z_{0}\right)$, it follows that $z_{0}^{(\tau)}$ has the order of inseparability $p^{e}$ over the field $K\left(z^{(1)}, \cdots, z^{(r-1)} ; z_{1}^{(r)}, \cdots, z_{n}^{(r)}\right)$. Denote by $K^{*}$ the field obtained from $K$ by the adjunction of the $n r$ element $z_{j}^{(i)}, i=1, \cdots, r$, $j=1, \cdots, n$. Then our result shows that $K\left(z^{(1)}, \cdots, z^{(r)}\right)$ has the order of inseparability not greater than $p^{\circ}$ over $K^{*}$. Consider now the form $F\left(Z, z^{(1)}, \cdots, z^{(r)}\right)$; it dissolves over the algebraic closure of $K\left(z^{(1)}, \cdots, z^{(r)}\right)$ into a product of distinct linear forms eách of which corresponds to an intersection of $U$ with the linear variety defined by the equations $\sum_{j=0}^{n} z_{j}^{(i)} X_{j}=0, i=1, \cdots, r$. For, the point $(x)$ is a simple point of $U$ (by the definition of a divisor) and the linear variety is a generic one containing the point $(x)$. Since the point $(x)$ is one of these intersections, therefore the linear form $\sum_{j=0}^{n} x_{j} Z_{j}$ is a simple factor of the form $F\left(Z, z^{(1)}, \cdots, z^{(r)}\right)$; it is, in fact, the only factor independent of the variables $z_{j}^{(i)}, i=1, \cdots, r ; j=1, \cdots, n$. Therefore, the point $(x)$ is rational over the field $K\left(z^{(1)}, \cdots, z^{(r)}\right)$, and hence $K^{*}(x)=K\left(z^{(1)}, \cdots, z^{(r)}\right)$. This shows that $K^{*}(x)$ has the order of inseparability not greater than $p^{e}$ over $K^{*}$; and since $K(x)$ and $K^{*}$ are linearly disjoint over $K$, it follows that $K(x)$ has also the order of inseparability not greater than $p^{e}$ over $K$.

The Johns Hopkins University 